\title{
Maximum Principle and the Applications of Mean-Field Backward Doubly Stochastic System
}

\author{
Hong Zhang ${ }^{1}$, Jingyi Wang ${ }^{2}$, Tengyu Zhao ${ }^{3}$, Li Zhou ${ }^{1}$ \\ ${ }^{1}$ School of Information, Beijing Wuzi University, Beijing, China \\ ${ }^{2}$ School of Banking and Finance, University of International Business and Economics, Beijing, China \\ ${ }^{3}$ School of Management Science and Engineering, Central University of Finance and Economics, Beijing, China
}

\section{Email address}

dr.yuwenjunxian@gmail.com (Hong Zhang)

\section{To cite this article:}

Hong Zhang, Jingyi Wang, Tengyu Zhao, Li Zhou. Maximum Principle and the Applications of Mean-Field Backward Doubly Stochastic System. Pure and Applied Mathematics Journal. Vol. 4, No. 3, 2015, pp. 101-108. doi: 10.11648/j.pamj.20150403.17

\begin{abstract}
Since Pardoux and Peng firstly studied the following nonlinear backward stochastic differential equations in 1990. The theory of BSDE has been widely studied and applied, especially in the stochastic control, stochastic differential games, financial mathematics and partial differential equations. In 1994, Pardoux and Peng came up with backward doubly stochastic differential equations to give the probabilistic interpretation for stochastic partial differential equations. Backward doubly stochastic differential equations theory has been widely studied because of its importance in stochastic partial differential equations and stochastic control problems. In this article, we will study the theory of doubly stochastic systems and related topics further.
\end{abstract}

Keywords: Mean-Field Backward Doubly, Stochastic System, Stochastic Control

\section{Introduction}

Andersson and Djehiche, Buckdahn, Djehiche and Li, Meyer Brandis, ksendal and Zhou, and Lihave studied the optimal control problem about Mean-field $\mathrm{s}$ tochastic differential system .Inspired by the above problems, in the paper, we study the optimal control problem about Mean-field backward doubly stochastic system. In the situation that control field to the convex and coefficient contains control variable, Using convex variational and dual technology, we present the local and global stochastic maximum principle, proved a sufficient conditions of optimality (verification theorem) and a necessary condition[1-4].

\section{The Control Problem of Mean-Field Backward Doubly Stochastic System}

For simple marking, make $m=n=d=l=k_{1}=k_{2}=1$. Given convex subset $U \subset \mathbb{R}^{k}$, allowing the control set is defined as

$$
\begin{gathered}
u_{a d}=\left\{v:[0, T] \times \Omega \rightarrow U \mid v \text { is } F_{t}\right. \text { - measurable, } \\
\left.\mathrm{E} \int_{o}^{T}|v(t)|^{2} d t<+\infty\right\}
\end{gathered}
$$

For any $v \in u_{a d}, \xi \in L^{2}\left(\Omega, F_{T}, P ; \mathrm{R}\right), \quad$ consider the following MF - BDSDE:

$$
Y^{v}(t)=\xi+\int_{t}^{T} \Gamma^{f}\left(s, Y^{v}(s), Z^{v}(s), v(s)\right) d s-\int_{t}^{T} Z^{v}(s) \vec{d} W(s)+\int_{t}^{T} \Gamma^{g}\left(s, Y^{v}(s), Z^{v}(s), v(s)\right) \overleftarrow{d} B(s)
$$

Where $i=f, g$

$$
\Gamma^{i}\left(s, Y^{v}(s), Z^{v}(s), v(s)\right)=\int_{\Omega} \theta^{i}\left(s, \omega, \omega^{\prime}, Y^{v}(s, \omega), Z^{v}(s, \omega), v(s, \omega), Y^{v}\left(s, \omega^{\prime}\right), Z^{v}\left(s, \omega^{\prime}\right), v\left(s, \omega^{\prime}\right)\right) P\left(d \omega^{\prime}\right),
$$

And $\theta^{f}: \Omega^{2} \times[0, T] \times \mathrm{R} \times \mathrm{R} \times U \times \mathrm{R} \times \mathrm{R} \times U \rightarrow \mathrm{R}, \theta^{g}: \Omega^{2} \times[0, T] \times \mathrm{R} \times \mathrm{R} \times U \times \mathrm{R} \times \mathrm{R} \times U \rightarrow \mathrm{R}$ 
Performance indicators is

$$
J(v(\cdot))=\mathrm{E} \int_{0}^{T} \Gamma^{l}\left(s, Y^{v}(s), Z^{v}(s), v(s)\right) d s+\mathrm{E}\left[\mathrm{E}^{\prime} h\left(Y_{0}^{v}(\omega), Y_{0}^{v}\left(\omega^{\prime}\right)\right)\right]
$$

Where

$$
\Gamma^{l}\left(s, Y^{v}(s), Z^{v}(s), v(s)\right)=\int_{\Omega} l\left(s, \omega, \omega^{\prime}, Y^{v}(s, \omega), Z^{v}(s, \omega), v(s, \omega), Y^{v}\left(s, \omega^{\prime}\right), Z^{v}\left(s, \omega^{\prime}\right), v\left(s, \omega^{\prime}\right)\right) P\left(d \omega^{\prime}\right)
$$

$h: \Omega^{2} \times \mathrm{R} \times \mathrm{R} \rightarrow \mathrm{R}$,

$l: \Omega^{2} \times[0, T] \times \mathrm{R} \times \mathrm{R} \times U \times \mathrm{R} \times \mathrm{R} \times U \rightarrow R$,

Control problem is looking for admission control to make performance indicators reaching the minimum value on the $u_{a d}$. Supposing that [5-6]

(H1) (1) $\theta^{f}, \theta^{g}, l, h$ is continuously differentiable about $y, y^{\prime}, z, z^{\prime}, v, v^{\prime}$, and the derivative of $h$ and $\mathrm{i}$ is linear growth.

(2) $\theta^{f}, \theta^{g}$ meet uniform Lipschitz condition about $\left(y, z, y^{\prime}, z^{\prime}, v, v^{\prime}\right)$.

In other words there exist $L_{i}, K_{i}, \alpha_{j}$, for $i=y, z, y^{\prime}, z^{\prime}, v, v^{\prime}, j=3,4$. making

$$
\begin{gathered}
\left|\theta^{f}\left(t, \omega, \omega^{\prime}, y_{1}, z_{1}, y_{1}^{\prime}, z_{1}^{\prime}, v_{1}, v_{1}^{\prime}\right)-\theta^{f}\left(t, \omega, \omega^{\prime}, y_{2}, z_{2}, y_{2}^{\prime}, z_{2}^{\prime}, v_{2}, v_{2}^{\prime}\right)\right| \\
\leq L_{y}\left|y_{1}-y_{2}\right|+L_{z}\left|z_{1}-z_{2}\right|+L_{y^{\prime}}\left|y_{1}^{\prime}-y_{2}^{\prime}\right|+L_{z^{\prime}}\left|z_{1}^{\prime}-z_{2}^{\prime}\right|+L_{v}\left|v_{1}-v_{2}\right|+L_{v^{\prime}}\left|v_{1}^{\prime}-v_{2}^{\prime}\right|, \\
\left|\theta^{g}\left(t, \omega, \omega^{\prime}, y_{1}, z_{1}, y_{1}^{\prime}, z_{1}^{\prime}, v_{1}, v_{1}^{\prime}\right)-\theta^{g}\left(t, \omega, \omega^{\prime}, y_{2}, z_{2}, y_{2}^{\prime}, z_{2}^{\prime}, v_{2}, v_{2}^{\prime}\right)\right|^{2} \\
\leq K_{y}^{2}\left|y_{1}-y_{2}\right|^{2}+K_{y^{\prime}}^{2}\left|y_{1}^{\prime}-y_{2}^{\prime}\right|^{2}+K_{v}^{2}\left|v_{1}-v_{2}\right|^{2}+K_{v^{\prime}}^{2}\left|v_{1}^{\prime}-v_{2}^{\prime}\right|^{2}+\alpha_{3}\left|z_{1}-z_{2}\right|+\alpha_{4}\left|z_{1}^{\prime}-z_{2}^{\prime}\right|, \\
\forall\left(t, \omega, \omega^{\prime}\right) \in[0, T] \times \Omega^{2},\left(y_{i}, z_{i}, y_{i}^{\prime}, z_{i}^{\prime}, v_{i}, v_{i}^{\prime}\right) \in \mathrm{R}^{6}, i=1,2
\end{gathered}
$$

And $\quad \mathrm{E} \int_{0}^{T}\left|\mathrm{E}^{\prime} \theta_{o}^{l}\left(t, \omega, \omega^{\prime}\right)\right|^{2} d t<\infty, \quad l=f, g$

Where $\theta_{0}^{l}\left(t, w, w^{\prime}\right)=\theta_{0}^{l}\left(t, w, w^{\prime}, 0,0,0,0,0,0\right), \alpha_{3}+\alpha_{4}<1$.

Under the above assumptions, for any $v(\bullet) \in u_{\text {ad }}$, there exists a unique solution $\left(Y^{v}, Z^{v}\right) \in S^{2}(0, T ; \mathbb{R}) \times M^{2}(0, T ; \mathbb{R})$ of the equation (1). And the performance index defined is reasonable.[7-8]

Assumed $\widehat{u}(\cdot)$ is the optimal control. $(\widehat{Y}(\cdot), \hat{Z}(\cdot))$ is the corresponding optimal trajectory. $v(\cdot)$ meet $\widehat{u}(\cdot)+v(\cdot) \in$ $u_{a d}$.because of the convexity of $u_{a d}$, for any

$$
\begin{gathered}
Y^{\varepsilon}(t)-\hat{Y}(t)=\int_{t}^{T}\left[\Gamma^{f}\left(s, Y^{\varepsilon}(s), Z^{\varepsilon}(s), u^{\varepsilon}(s)\right)-\Gamma^{f}(s, \hat{Y}(s), \hat{Z}(s), \hat{u}(s))\right] d s \\
+\int_{t}^{T}\left[\Gamma^{g}\left(s, Y^{\varepsilon}(s), Z^{\varepsilon}(s), u^{\varepsilon}(s)\right)-\Gamma^{g}(s, \hat{Y}(s), \hat{Z}(s), \hat{u}(s))\right] \stackrel{\leftarrow}{d} B(s)-\int_{t}^{T}\left(Z^{\varepsilon}(s)-\hat{Z}(s)\right) \vec{d} W(s)
\end{gathered}
$$

Applying Itô formulas to $\left|Y^{\mathcal{E}}(t)-\hat{Y}(t)\right|^{2}$

$$
\begin{gathered}
E\left(\left|Y^{\varepsilon}(t)-\hat{Y}(t)\right|^{2}+\int_{t}^{T}\left|Z^{\varepsilon}(s)-\hat{Z}(s)\right|^{2} d s\right)=2 \mathrm{E} \int_{t}^{T}\left\langle Y^{\varepsilon}(s)-\hat{Y}(s), \Gamma^{f}\left(s, Y^{\varepsilon}(s), Z^{\varepsilon}(s), u^{\varepsilon}(s)\right)-\Gamma^{f}(s, \hat{Y}(s), \hat{Z}(s), \hat{u}(s))\right\rangle d s \\
+\mathrm{E} \int_{t}^{T}\left|\Gamma^{g}\left(s, Y^{\varepsilon}(s), Z^{\varepsilon}(s), u^{\varepsilon}(s)\right)-\Gamma^{g}(s, \hat{Y}(s), \hat{Z}(s), \hat{u}(s))\right|^{2} d s
\end{gathered}
$$

According to (H1), there is 


$$
\mathrm{E}\left|Y^{\mathcal{E}}(t)-\hat{Y}(t)\right|^{2}+\mathrm{E} \int_{t}^{T}\left|Z^{\mathcal{E}}(s)-\hat{Z}(s)\right|^{2} d s \leq k_{1} \mathrm{E} \int_{t}^{T}\left|Y^{\mathcal{E}}(t)-\hat{Y}(t)\right|^{2} d s+k_{2} \varepsilon^{2} \mathrm{E} \int_{t}^{T}|v(s)|^{2} d s
$$

Where $k_{i}(i=1,2)$ is constant rely on (H1). According Gronwall Inequality and Burkholder-Davis-Gundy Inequality, results are verified.

For simple marking, make

$$
\begin{gathered}
\hat{\alpha}(\cdot)=\alpha\left(\cdot, \omega, \omega^{\prime}, \hat{Y}(\cdot, \omega), \hat{Z}(\cdot, \omega), \hat{u}(\cdot, \omega), \hat{Y}\left(\cdot, \omega^{\prime}\right), \hat{Z}\left(\cdot, \omega^{\prime}\right), \hat{u}\left(\cdot, \omega^{\prime}\right)\right), \\
\alpha^{\varepsilon}(\cdot)=\alpha\left(\cdot, \omega, \omega^{\prime}, Y^{\varepsilon}(\cdot, \omega), Z^{\varepsilon}(\cdot, \omega), u^{\varepsilon}(\cdot, \omega), Y^{\varepsilon}\left(\cdot, \omega^{\prime}\right), Z^{\varepsilon}\left(\cdot, \omega^{\prime}\right), u^{\varepsilon}\left(\cdot, \omega^{\prime}\right)\right), \\
\xi(t)=\psi(t)+\int_{t}^{T} F_{1}(s, \xi(s), \eta(s)) d s+\int_{t}^{T} G_{1}(s, \xi(s), \eta(s)) \bar{d} B(s)-\int_{t}^{T} \eta(s) \vec{d} W(s),
\end{gathered}
$$

Where

$$
\begin{aligned}
& F_{1}(s, \xi(s), \eta(s))=\mathrm{E}^{\prime}\left[{\widehat{\theta^{f}}}_{y}(s) \xi(s)+{\widehat{\theta^{f}}}_{z}(s) \eta(s)+{\widehat{\theta^{f}}}_{y^{\prime}}(s) \xi^{\prime}(s)+{\widehat{\theta^{f}}}_{z^{\prime}}(s) \eta^{\prime}(s)\right], \\
& G_{1}(s, \xi(s), \eta(s))=\mathrm{E}^{\prime}\left[{\widehat{\theta^{g}}}_{y}(s) \xi(s)+{\widehat{\theta^{g}}}_{z}(s) \eta(s)+{\widehat{\theta^{g}}}_{y^{\prime}}(s) \xi^{\prime}(s)+{\widehat{\theta^{g}}}_{z^{\prime}}(s) \eta^{\prime}(s)\right],
\end{aligned}
$$

And

$$
\psi(t)=\int_{t}^{T} \mathrm{E}^{\prime}\left[{\widehat{\theta^{f}}}_{v}(s) v(s)+{\widehat{\theta^{f}}}_{v^{\prime}}(s) v^{\prime}(s)\right] d s+\int_{t}^{T} \mathrm{E}^{\prime}\left[{\widehat{\theta^{g}}}_{v}(s) v(s)+{\widehat{\theta^{g}}}_{v^{\prime}}(s) v^{\prime}(s)\right] \bar{d} B(s)
$$

Marked

$$
\begin{aligned}
\mathrm{E}^{\prime}\left[{\widehat{\theta^{f}}}_{y}(s) \xi(s)\right] & =\int_{\Omega}{\widehat{\theta^{f}}}_{y}\left(s, \omega, \omega^{\prime}\right) \xi(s, \omega) \mathrm{P}\left(d \omega^{\prime}\right), \\
\mathrm{E}^{\prime}\left[{\widehat{\theta^{f}}}_{y^{\prime}}(s) \xi^{\prime}(s)\right] & =\int_{\Omega}{\widehat{\theta^{f}}}_{y^{\prime}}\left(s, \omega, \omega^{\prime}\right) \xi\left(s, \omega^{\prime}\right) \mathrm{P}\left(d \omega^{\prime}\right) .
\end{aligned}
$$

Under the above assumptions, for any $v(\bullet) \in u_{a d}$, there exists a unique solution $(\xi(t), \eta(t)) \in S^{2}([0, T] ; \mathrm{R}) \times M^{2}(0, T ; \mathbb{R})$ of the equation (2).

Lemma 2. Marked

$$
\begin{gathered}
y^{\varepsilon}(t)=\frac{Y^{\varepsilon}(t)-\hat{Y}(t)}{\mathcal{E}}-\xi(t), \quad z^{\varepsilon}(t)=\frac{Z^{\varepsilon}(t)-\hat{Z}(t)}{\mathcal{E}}-\eta(t) . \\
\lim _{\varepsilon \rightarrow 0} \sup _{t \in[0, T]} \mathrm{E}\left|y^{\varepsilon}(t)\right|^{2}=0, \quad \lim _{\varepsilon \rightarrow 0} \mathrm{E} \int_{0}^{T}\left|z^{\varepsilon}(t)\right|^{2} d t=0 .
\end{gathered}
$$

$\left(y^{\varepsilon}, z^{\varepsilon}\right)$ is the solution of the equation as follows,

$$
\left\{\begin{aligned}
-d y^{\varepsilon}(t)= & \mathrm{E}^{\prime}\left[f_{y}^{\varepsilon}(t) y^{\varepsilon}(t)+f_{z}^{\varepsilon}(t) z^{\varepsilon}(t)+f_{y^{\prime}}^{\varepsilon}(t) y^{\prime \varepsilon}(t)+f_{z^{\prime}}^{\varepsilon}(t) z^{\prime \varepsilon}(t)+f_{1}^{\varepsilon}(t)\right] d t \\
& +\mathrm{E}^{\prime}\left[g_{y}^{\varepsilon}(t) y^{\varepsilon}(t)+g_{z}^{\varepsilon}(t) z^{\varepsilon}(t)+g_{y^{\prime}}^{\varepsilon}(t) y^{\prime \varepsilon}(t)+g_{z^{\prime}}^{\varepsilon}(t) z^{\varepsilon}(t)+g_{1}^{\varepsilon}(t)\right] \bar{d} B(t) \\
& -z^{\varepsilon}(t) \vec{d} W(t), \\
y^{\varepsilon}(T)=0, &
\end{aligned}\right.
$$

Where $\delta=f, g, \quad \bar{Y}_{t, \omega}=\hat{Y}_{t, \omega}+\lambda\left(Y_{t, \omega}^{\varepsilon}-\hat{Y}_{t, \omega}\right), \quad \bar{u}_{t, \omega}=\hat{u}_{t, \omega}+\lambda\left(u_{t, \omega}^{\varepsilon}-\hat{u}_{t, \omega}\right)$

$$
\delta_{y}^{\varepsilon}(\cdot)=\int_{0}^{1} \theta_{y}^{\delta}\left(\cdot, \bar{Y}_{;, \omega}, \bar{Z}_{;, \omega}, \bar{u}_{, \omega}, \bar{Y}_{;, \omega^{\prime}}, \bar{Z}_{;, \omega^{\prime}}, \bar{u}_{\cdot, \omega^{\prime}}\right) d \lambda
$$


and

$$
\begin{gathered}
\delta_{1}^{\varepsilon}(\cdot)=\left[\delta_{y}^{\varepsilon}(\cdot)-\widehat{\theta}_{y}^{\delta}(\cdot)\right] \xi_{;, \omega}+\left[\delta_{z}^{\varepsilon}(\cdot)-\widehat{\theta}_{z}^{\delta}(\cdot)\right] \eta_{, \omega}+\left[\delta_{y^{\prime}}^{\varepsilon}(\cdot)-\widehat{\theta}_{y^{\prime}}^{\delta}(\cdot)\right] \xi_{;, \omega^{\prime}} \\
+\left[\delta_{z^{\prime}}^{\varepsilon}(\cdot)-\widehat{\theta}_{z^{\prime}}^{\delta}(\cdot)\right] \eta_{, \omega^{\prime \prime}}+\left[\delta_{v}^{\varepsilon}(\cdot)-\widehat{\theta}_{v}^{\delta}(\cdot)\right] v_{\cdot, \omega}+\left[\delta_{v^{\prime}}^{\varepsilon}(\cdot)-\widehat{\theta}_{v^{\prime}}(\cdot)\right] v_{;, \omega^{\prime}}
\end{gathered}
$$

Applying Itô formulas to $\left|y^{\varepsilon}(t)\right|^{2}$ on $[t, T]$

$$
\begin{gathered}
\mathrm{E}\left|y^{\varepsilon}(t)\right|^{2}+\mathrm{E} \int_{t}^{T}\left|z^{\varepsilon}(s)\right|^{2} d s=2 \mathrm{E} \int_{t}^{T}\left\langle y^{\varepsilon}(s), \mathrm{E}^{\prime}\left[f_{y}^{\varepsilon}(s) y^{\varepsilon}(s)+f_{z}^{\varepsilon}(s) z^{\varepsilon}(s)+f_{y^{\prime}}^{\varepsilon}(s) y^{\prime \varepsilon}(s)+f_{z^{\prime}}^{\varepsilon}(s) z^{\prime \varepsilon}(s)+f_{1}^{\varepsilon}(s)\right]\right\rangle d s \\
+\mathrm{E} \int_{t}^{T}\left|\mathrm{E}^{\prime}\left[g_{y}^{\varepsilon}(s) y^{\varepsilon}(s)+g_{z}^{\varepsilon}(s) z^{\varepsilon}(s)+g_{y^{\prime}}^{\varepsilon}(s) y^{\prime}(s)+g_{z^{\prime}}^{\varepsilon}(s) z^{\varepsilon}(s)+g_{1}^{\varepsilon}(s)\right]\right|^{2} d s .
\end{gathered}
$$

According to (H1), there is

$$
\mathrm{E}\left|y^{\varepsilon}(t)\right|^{2}+\mathrm{E} \int_{t}^{T}\left|z^{\varepsilon}(s)\right|^{2} d s \leq k \mathrm{E} \int_{t}^{T}\left|y^{\varepsilon}(s)\right|^{2} d s+C_{\varepsilon},
$$

Where $k$ is constant, when $\varepsilon \rightarrow 0$ 时, $C_{\varepsilon} \rightarrow 0$. According Gronwall Inequality, results are verified. Because of $\widehat{u}(\cdot)$ is the optimal control,

$$
\mathcal{E}^{-1}\left[J\left(u^{\varepsilon}(\cdot)\right)-J(\hat{u}(\cdot))\right] \geq 0 .
$$

According to lemma 2, there is

lemma 3. Hypothesis (H1) was established, then the following variation inequality is established ${ }^{[9-10]}$ :

$$
\begin{gathered}
\mathrm{E}_{0}^{T} \mathrm{E}^{\prime}\left[\hat{l}_{y}(s) \xi(s)+\hat{l}_{z}(s) \eta(s)+\hat{l}_{y^{\prime}}(s) \xi^{\prime}(s)+\hat{l}_{z^{\prime}}(s) \eta^{\prime}(s)+\hat{l}_{v}(s) v(s)+\hat{l}_{v^{\prime}}(s) v^{\prime}(s)\right] d s \\
+\mathrm{EE}^{\prime}\left[h_{y}\left(\hat{Y}_{0, \omega}, \hat{Y}_{0, \omega^{\prime}}\right) \xi_{0, \omega}+h_{y^{\prime}}\left(\hat{Y}_{0, \omega}, \hat{Y}_{0, \omega^{\prime}}\right) \xi_{0, \omega^{\prime}}\right] \geq 0 .
\end{gathered}
$$

Where

$$
\mathrm{E}^{\prime}\left[\hat{l}_{y^{\prime}}(s) \xi^{\prime}(s)\right]=\int_{\Omega} \hat{l}_{y^{\prime}}\left(s, \omega, \omega^{\prime}\right) \xi\left(s, \omega^{\prime}\right) \mathrm{P}\left(d \omega^{\prime}\right) .
$$

Proof.

$$
\begin{gathered}
\mathrm{EE}^{\prime} \varepsilon^{-1}\left[h\left(Y_{0, \omega}^{\varepsilon}, Y_{0, \omega^{\prime}}^{\varepsilon}\right)\right]-h\left(\hat{Y}_{0, \omega}, \hat{Y}_{0, \omega^{\prime}}\right)=\mathrm{EE}^{\prime} \varepsilon^{-1} \int_{0}^{1} h_{y}\left(\bar{Y}_{0, \omega}, \bar{Y}_{0, \omega^{\prime}}\right)\left(Y_{0, \omega}^{\varepsilon}-\hat{Y}_{0, \omega}\right) d \lambda+\mathrm{EE}^{\prime} \varepsilon^{-1} \int_{0}^{1} h_{y^{\prime}}\left(\bar{Y}_{0, \omega}, \bar{Y}_{0, \omega^{\prime}}\right)\left(Y_{0, \omega^{\prime}}^{\varepsilon}-\hat{Y}_{0, \omega^{\prime}}\right) d \lambda \\
\rightarrow \mathrm{EE}^{\prime}\left[h_{y}\left(\hat{Y}_{0, \omega}, \hat{Y}_{0, \omega^{\prime}}\right) \xi_{0, \omega}+h_{y^{\prime}}\left(\hat{Y}_{0, \omega}, \hat{Y}_{0, \omega^{\prime}}\right) \xi_{0, \omega^{\prime}}\right], \varepsilon \rightarrow 0
\end{gathered}
$$

Where $\bar{Y}_{0, \omega}=\hat{Y}_{0, \omega}+\lambda\left(Y_{0, \omega}^{\varepsilon}-\hat{Y}_{0, \omega}\right)$.

$$
\varepsilon^{-1}\left\{\mathrm{E} \int_{0}^{T} \mathrm{E}^{\prime}\left[l^{\varepsilon}(t)-\hat{l}(t)\right] d t\right\} \rightarrow \mathrm{E} \int_{0}^{T} \mathrm{E}^{\prime}\left[\hat{l}_{y}(s) \xi(s)+\hat{l}_{z}(s) \eta(s)+\hat{l}_{y^{\prime}}(s) \xi^{\prime}(s)+\hat{l}_{z^{\prime}}(s) \eta^{\prime}(s)+\hat{l}_{u}(s) v(s)+\hat{l}_{v^{\prime}}(s) v^{\prime}(s)\right] d s
$$

so (5) is verified.

Considering the adjoin equation:

$$
p(t)=\mathrm{E}^{\prime} h_{y}\left(\hat{Y}_{0, \omega}, \hat{Y}_{0, \omega^{\prime}}\right)+\mathrm{E}^{*} h_{y^{\prime}}\left(\hat{Y}_{0, \omega^{*}}, \hat{Y}_{0, \omega}\right)+\int_{0}^{t} F_{2}(s, p(s), q(s)) d s+\int_{0}^{t} G_{2}(s, p(s), q(s)) \vec{d} W(s)-\int_{0}^{t} q(s) \bar{d} B(s),
$$

Where

$$
F_{2}(s, p(s), q(s))=\mathrm{E}^{\prime}\left[{\widehat{\theta^{f}}}_{y}(s) p(s)+{\widehat{\boldsymbol{\theta}^{g}}}_{y}(s) q(s)+\hat{l}_{y}(s)\right]+\mathrm{E}^{*}\left[{\widehat{\boldsymbol{\theta}^{f}}}_{y^{\prime}}(s) p^{*}(s)+{\widehat{\boldsymbol{\theta}^{g}}}_{y^{\prime}}(s) q^{*}(s)+\hat{l}_{y^{\prime}}(s)\right],
$$




$$
\begin{gathered}
G_{2}(s, p(s), q(s))=\mathrm{E}^{\prime}\left[\widehat{\theta}^{f}{ }_{z}(s) p(s)+{\widehat{\theta^{g}}}_{z}(s) q(s)+\hat{l}_{z}(s)\right]+\mathrm{E}^{*}\left[\widehat{\theta^{f}} z^{\prime}(s) p^{*}(s)+{\widehat{\theta^{g}}}_{z^{\prime}}(s) q^{*}(s)+\widehat{l_{z^{\prime}}}(s)\right] . \\
\mathrm{E}^{*} \hat{l}_{y^{\prime}}(s)=\int_{\Omega} \hat{l}_{y^{\prime}}\left(s, \omega^{*}, \omega\right) \mathrm{P}\left(d \omega^{*}\right), \\
\mathrm{E}^{*}\left[\widehat{\theta}_{y^{\prime}}(s) p^{*}(s)\right]=\int_{\Omega} \widehat{\theta^{f}} y^{\prime}\left(s, \omega^{*}, \omega\right) p\left(s, \omega^{*}\right) \mathrm{P}\left(d \omega^{*}\right) .
\end{gathered}
$$

Define the Hamiltonian function $H:[0, T] \times R \times R \times R \times R \times R \times R \times R \times R \rightarrow R$ as follows

$$
\begin{aligned}
& H\left(t, y_{1}, z_{1}, v_{1}, y_{2}, z_{2}, v_{2}, p, q\right)=\theta^{f}\left(t, \omega, \omega^{\prime}, y_{1}, z_{1}, v_{1}, y_{2}, z_{2}, v_{2}\right) p+\theta^{g}\left(t, \omega, \omega^{\prime}, y_{1}, z_{1}, v_{1}, y_{2}, z_{2}, v_{2}\right) q \\
&+l\left(t, \omega, \omega^{\prime}, y_{1}, z_{1}, v_{1}, y_{2}, z_{2}, v_{2}\right)
\end{aligned}
$$

By the variational inequality (7), we present MF - BDSDEs stochastic control problem of stochastic maximum principle.

Theorem 1,(stochastic maximum principle) Assumed $(\hat{Y}(\cdot), \hat{Z}(\cdot), \hat{u}(\cdot))$ is the optimal trajectory of the control problem $\{(1),(2)\}, \forall v \in U$, a.e. $\quad t \in[0, T]$, a.s.

$$
\left[\mathrm{E}^{\prime} \widehat{H}_{v}\left(t, \omega, \omega^{\prime}\right)+\mathrm{E}^{*} H_{v^{\prime}}\left(t, \omega^{*}, \omega\right)\right] \cdot(v-\hat{u}(t)) \geq 0
$$

where

$$
\widehat{H}\left(t, \omega, \omega^{\prime}\right)=H\left(t, \omega, \omega^{\prime}, \hat{Y}(t, \omega), \hat{Z}(t, \omega), \hat{u}(t, \omega), \hat{Y}\left(t, \omega^{\prime}\right), \hat{Z}\left(t, \omega^{\prime}\right), \hat{u}\left(t, \omega^{\prime}\right), p(t, \omega), q(t, \omega)\right)
$$

Proof. Applying Itô formulas to $\langle\xi(t), p(t)\rangle$,we can get

$$
\begin{gathered}
-\mathrm{E} \xi_{0} p_{0}=\mathrm{E} \int_{0}^{T} \mathrm{E}^{\prime}\left[l_{y}(s) \xi(s)+l_{z}(s) \eta(s)+l_{y^{\prime}}(s) \xi^{\prime}(s)+l_{z^{\prime}}(s) \eta^{\prime}(s)\right] d s-\mathrm{E} \int_{0}^{T} \mathrm{E}^{\prime}\left[\widehat{\theta^{f}} v^{\prime}(s) v^{\prime}(s) p(s)+{\widehat{\theta^{g}}}_{v^{\prime}}(s) v^{\prime}(s) q(s)\right] d s \\
-\mathrm{E} \int_{0}^{T} \mathrm{E}^{\prime}\left[\widehat{\theta}^{f}{ }_{v}(s) v(s) p(s)+\widehat{\theta}^{g}{ }_{v}(s) v(s) q(s)\right] d s
\end{gathered}
$$

According (5), we can get

$$
\begin{aligned}
& \mathrm{E} \int_{0}^{T} \mathrm{E}^{\prime}\left[\widehat{\boldsymbol{\theta}}^{f} v^{\prime}(s) v^{\prime}(s) p(s)+{\widehat{\theta^{g}}}_{v^{\prime}}(s) v^{\prime}(s) q(s)+\hat{l}_{v^{\prime}}(s) v^{\prime}(s)\right] d s \\
& \mathrm{E} \int_{0}^{T} \mathrm{E}^{\prime}\left[{\widehat{\theta^{f}}}_{v}(s) v(s) p(s)+{\widehat{\boldsymbol{\theta}^{g}}}_{v}(s) v(s) q(s)+\hat{l}_{v}(s) v(s)\right] d s \geq 0
\end{aligned}
$$

According Hamiltonian function, we can get

$$
\begin{gathered}
\mathrm{E} \int_{0}^{T}\left[\mathrm { E } ^ { * } H _ { v ^ { \prime } } \left(t, \hat{Y}(t)\left(\omega^{*}\right), \hat{Z}(t)\left(\omega^{*}\right), \hat{u}(t)\left(\omega^{*}\right), \hat{Y}(t, \omega), \hat{Z}(t, \omega), \hat{u}(t, \omega),\right.\right. \\
\left.\left.p(t)\left(\omega^{*}\right), q(t)\left(\omega^{*}\right)\right)+\mathrm{E}^{\prime} H_{v}\left(t, \hat{Y}(t, \omega), \hat{Z}(t, \omega), \hat{u}(t, \omega), \hat{Y}(t)\left(\omega^{\prime}\right), \hat{Z}(t)\left(\omega^{\prime}\right), \hat{u}(t)\left(\omega^{\prime}\right), p(t, \omega), q(t, \omega)\right)\right] \cdot v(t) d t \geq 0
\end{gathered}
$$

For any $v \in U, F$ is the any element of $\sigma-A \lg e b r a\left(F_{t}\right)$,setting

$$
\bar{v}(s)= \begin{cases}\hat{u}(s), & s \in[0, t), \\ v, & s \in[t,, t+\varepsilon), \omega \in F, \\ \hat{u}(s), & s \in[t,, t+\varepsilon), \omega \in \Omega-F, \\ \hat{u}(s), & s \in[t+\varepsilon, T],\end{cases}
$$

We can know $v(s) \in u_{a d}$, because $v(t)$ meet $\hat{u}(t)+v(t) \in u_{a d}$, setting $v(t)=\bar{v}(t)-\hat{u}(t)$, The above inequalities can be rewritten as 


$$
\mathrm{El}_{F} \int_{t}^{t+\varepsilon}\left[\mathrm{E}^{\prime} \widehat{H}_{v}\left(s, \omega, \omega^{\prime}\right)+\mathrm{E}^{*} \widehat{H}_{v^{\prime}}\left(s, \omega^{*}, \omega\right)\right] \cdot(v-\hat{u}(s)) d s \geq 0
$$

Differential on a variable $\varepsilon$ at $\varepsilon=0$, we can get

$$
\mathrm{El}_{F}\left[\mathrm{E}^{\prime} \widehat{H}_{v}\left(t, \omega, \omega^{\prime}\right)+\mathrm{E}^{*} \widehat{H}_{v^{\prime}}\left(t, \omega^{*}, \omega\right)\right] \cdot(v-\hat{u}(t)) \geq 0
$$

So (8) is verified.

\section{Mean-Field Backward Doubly Stochastic LQ Problem}

This section, we apply the maximum value principle to Mean-field backward doubly stochastic LQ problem.

$$
q\left(Y_{0, \omega}, Y_{0, \omega^{\prime}}\right)=\frac{1}{2} Q_{0}^{1} Y_{0, \omega}^{2}+\frac{1}{2} Q_{0}^{2} Y_{0, \omega^{\prime}}^{2}
$$

And

$$
\begin{gathered}
f\left(s, \omega, \omega^{\prime}, Y_{s, \omega}, Z_{s, \omega}, v_{s, \omega}, Y_{s, \omega^{\prime}}, Z_{s, \omega^{\prime}}, v_{s, \omega^{\prime}}\right)=A^{1}(s) Y_{s, \omega}+B^{1}(s) Z_{s, \omega}+C^{1}(s) v_{s, \omega}+A^{2}(s) Y_{s, \omega^{\prime}}+B^{2}(s) Z_{s, \omega^{\prime}}+C^{2}(s) v_{s, \omega^{\prime}} \\
g\left(s, \omega, \omega^{\prime}, Y_{s, \omega}, Z_{s, \omega}, v_{s, \omega}, Y_{s, \omega^{\prime}}, Z_{s, \omega^{\prime}}, v_{s, \omega^{\prime}}\right)=D^{1}(s) Y_{s, \omega}+E^{1}(s) Z_{s, \omega}+F^{1}(s) v_{s, \omega}+D^{2}(s) Y_{s, \omega^{\prime}}+E^{2}(s) Z_{s, \omega^{\prime}}+F^{2}(s) v_{s, \omega^{\prime}} \\
l\left(s, \omega, \omega^{\prime}, Y_{s, \omega}, Z_{s, \omega}, v_{s, \omega}, Y_{s, \omega^{\prime}}, Z_{s, \omega^{\prime}}, v_{s, \omega^{\prime}}\right)=\frac{1}{2}\left[M^{1}(s) Y_{s, \omega}^{2}+N^{1}(s) Z_{s, \omega}^{2}+W^{1}(s) v_{s, \omega}^{2}+M^{2}(s) Y_{s, \omega^{\prime}}^{2}+N^{2}(s) Z_{s, \omega^{\prime}}^{2}+W^{2}(s) v_{s, \omega^{\prime}}^{2}\right]
\end{gathered}
$$

Where $A^{i}:[0, T] \times \Omega^{2} \rightarrow \mathbb{R}$ is bounded. $\left(s, w, w^{\prime}\right) \mapsto A^{i}\left(s, w, w^{\prime}\right)$ is $\mathfrak{M}_{s}^{2}$ measurable (similarly, other coefficient satisfies the hypothesis). $M^{i}, N^{i}$ are nonnegative, $R^{i}$ is positive. The state equation is

$$
\begin{gathered}
Y_{t, \omega}^{v}=\xi+\int_{t}^{T}\left\{\left[\mathrm{E}^{\prime} A^{1}(s)\right] Y_{s, \omega}^{v}+\left[\mathrm{E}^{\prime} B^{1}(s)\right] Z_{s, \omega}^{v}+\left[\mathrm{E}^{\prime} C^{1}(s)\right] v_{s, \omega}\right\} d s+\int_{t}^{T} \mathrm{E}^{\prime}\left[A^{2}(s) Y_{s, \omega^{\prime}}^{v}+B^{2}(s) Z_{s, \omega^{\prime}}^{v}+C^{2}(s) v_{s, \omega^{\prime}}\right] d s \\
+\int_{t}^{T}\left\{\left[\mathrm{E}^{\prime} D^{1}(s)\right] Y_{s, \omega}^{v}+\left[\mathrm{E}^{\prime} E^{1}(s)\right] Z_{s, \omega}^{v}+\left[\mathrm{E}^{\prime} F^{1}(s)\right] v_{s, \omega}\right\} \bar{d} B_{s, \omega}+\int_{t}^{T}\left\{\mathrm{E}^{\prime}\left[D^{2}(s) Y_{s, \omega^{\prime}}^{v}+E^{2}(s) Z_{s, \omega^{\prime}}^{v}+F^{2}(s) v_{s, \omega^{\prime}}\right]\right\} \bar{d} B_{s, \omega} \\
\quad-\int_{t}^{T} Z_{s, \omega}^{v} \vec{d} W_{s, \omega},
\end{gathered}
$$

Performance indicators is

$$
\begin{gathered}
J(v(\cdot))=\frac{1}{2} \mathrm{E}\left(\int_{0}^{T} \mathrm{E}^{\prime}\left[M^{1}(s)\left|Y_{s, \omega}^{v}\right|^{2}+N^{1}(s)\left|Z_{s, \omega}^{v}\right|^{2}+W^{1}(s) v_{s, \omega}^{2}\right] d s+\mathrm{E}^{\prime}\left[Q_{0}^{1}\left|Y_{0, \omega}^{v}\right|^{2}\right]\right) \\
\frac{1}{2} \mathrm{E}\left(\int_{0}^{T} \mathrm{E}^{\prime}\left[M^{2}(s)\left|Y_{s, \omega^{\prime}}^{v}\right|^{2}+N^{2}(s)\left|Z_{s, \omega^{\prime}}^{v}\right|^{2}+W^{2}(s)\left|v_{s, \omega^{\prime}}\right|^{2}\right] d s+\mathrm{E}^{\prime}\left[Q_{0}^{2}\left|Y_{0, \omega^{\prime}}^{v}\right|^{2}\right]\right) .
\end{gathered}
$$

In order to mark is simple, put $A^{i}\left(s . \omega, \omega^{\prime}\right)$ for $A^{i}(s)$. Hamiltonian function is

$$
\begin{gathered}
H\left(s, \omega, \omega^{\prime}, y_{1}, z_{1}, v_{1}, y_{2}, z_{2}, v_{2}, p, q\right)=\left[A^{1}(s) y_{1}+B^{1}(s) z_{1}+C^{1}(s) v_{1}+A^{2}(s) y_{2}+B^{2}(s) z_{2}+C^{2}(s) v_{2}\right] p \\
+\left[D^{1}(s) y_{1}+E^{1}(s) z_{1}+F^{1}(s) v_{1}+D^{2}(s) y_{2}+E^{2}(s) z_{2}+F^{2}(s) v_{2}\right] q \\
+\frac{1}{2}\left[M^{1}(s) y_{1}^{2}+N^{1}(s) z_{1}^{2}+W^{1}(s) v_{1}^{2}+M^{2}(s) y_{2}^{2}+N^{2}(s) z_{2}^{2}+W^{2}(s) v_{2}^{2}\right]
\end{gathered}
$$

According Theorem 1, we can get

$$
0=\mathrm{E}^{\prime}\left[C^{1}(s) p_{s}+F^{1}(s) q_{s}+W^{1}(s) \hat{u}_{s}\right]+\mathrm{E}^{*}\left[C^{2}(s) p_{s}^{*}+F^{2}(s) q_{s}^{*}+W^{2}(s) \hat{u}_{s}\right],
$$

Where 


$$
\begin{gathered}
\mathrm{E}^{*}\left[C^{1}(s) p_{s}\right]=\int_{\Omega} C^{1}\left(s, \omega, \omega^{\prime}\right) p(s, \omega) P\left(d \omega^{\prime}\right), \\
\mathrm{E}^{*}\left[W^{2}(s) \hat{u}(s)\right]=\int_{\Omega} W^{2}\left(s, \omega^{*}, \omega\right) u(s, \omega) P\left(d \omega^{*}\right), \\
\mathrm{E}^{*}\left[C^{2}(s) p_{s}^{*}\right]=\int_{\Omega} C^{2}\left(s, \omega^{*}, \omega\right) p\left(s, \omega^{*}\right) P\left(d \omega^{*}\right),
\end{gathered}
$$

And

$$
p(t)=\mathrm{E}^{\prime}\left[Q_{0}^{1} \hat{Y}_{0, \omega}\right]+\mathrm{E}^{*}\left[Q_{0}^{2} \hat{Y}_{0, \omega}\right]+\int_{0}^{t} F_{2}(s, p(s), q(s)) d s+\int_{0}^{t} G_{2}(s, p(s), q(s)) \vec{d} W(s)-\int_{0}^{t} q(s) \bar{d} B(s),
$$

And

$$
\begin{aligned}
& F_{2}(s, p(s), q(s))=\mathrm{E}^{\prime}\left[A^{1}(s) p(s)+D^{1}(s) q(s)+M^{1}(s)\right]+\mathrm{E}^{*}\left[A^{2}(s) p^{*}(s)+D^{2}(s) q^{*}(s)+M^{2}(s)\right] \\
& G_{2}(s, p(s), q(s))=\mathrm{E}^{\prime}\left[B^{1}(s) p(s)+E^{1}(s) q(s)+N^{1}(s)\right]+\mathrm{E}^{*}\left[B^{2}(s) p^{*}(s)+E^{2}(s) q^{*}(s)+N^{2}(s)\right]
\end{aligned}
$$

Theorem 2. Assumes $\hat{u}$ that satisfy (9), the (p,g) is the solution of equation (10), the above LQ problem have unique solution Proof.

$$
\begin{gathered}
J(v)-J(\hat{u})=\frac{1}{2} \mathrm{E}_{0}^{T} \mathrm{E}^{\prime}\left[M^{1}(s)\left(\left|Y_{s, \omega}^{v}\right|^{2}-\left|\hat{Y}_{s, \omega}\right|^{2}\right)+N^{1}(s)\left(\left|Z_{s, \omega}^{v}\right|^{2}-\left|\hat{Z}_{s, \omega}\right|^{2}\right)\right] d s \\
+\frac{1}{2} \mathrm{E} \int_{0}^{T} \mathrm{E}^{\prime}\left[W^{1}(s)\left(\left|v_{s, \omega}\right|^{2}-\left|\hat{u}_{s, \omega}\right|^{2}\right)+M^{2}(s)\left(\left|Y_{S, \omega^{\prime}}^{v}\right|^{2}-\left|\hat{Y}_{s, \omega^{\prime}}\right|^{2}\right)\right] d s \\
+\frac{1}{2} \mathrm{E} \int_{0}^{T} \mathrm{E}^{\prime}\left[N^{2}(s)\left(\left|Z_{s, \omega^{\prime}}^{v}\right|^{2}-\left|\hat{Z}_{s, \omega^{\prime}}\right|^{2}\right)+W^{2}(s)\left(\left|v_{s, \omega^{\prime}}\right|^{2}-\left|\hat{u}_{s, \omega^{\prime}}\right|^{2}\right)\right] d s+\frac{1}{2} \mathrm{E}^{\prime}\left[Q_{0}^{1}\left(\left|Y_{0, \omega}^{v}\right|^{2}-\left|\hat{Y}_{0, \omega}\right|^{2}\right)+Q_{0}^{2}\left(\left|Y_{0, \omega^{\prime}}^{v}\right|^{2}-\left|\hat{Y}_{0, \omega^{\prime}}\right|^{2}\right)\right] \\
\geq \mathrm{E} \int_{0}^{T} \mathrm{E}^{\prime}\left[M^{1}(s) \hat{Y}_{s, \omega}\left(Y_{s, \omega}^{v}-\hat{Y}_{s, \omega}\right)+N^{1}(s) \hat{Z}_{s, \omega}\left(Z_{s, \omega}^{v}-\hat{Z}_{s, \omega}\right)\right] d s+\mathrm{E} \int_{0}^{T} \mathrm{E}^{\prime}\left[W^{1}(s) \hat{u}_{s, \omega}\left(v_{s, \omega}-\hat{u}_{s, \omega}\right)+M^{2}(s) \hat{Y}_{s, \omega^{\prime}}\left(Y_{s, \omega^{\prime}}^{v}-\hat{Y}_{s, \omega^{\prime}}\right)\right] d s \\
+\mathrm{E} \int_{0}^{T} \mathrm{E}^{\prime}\left[N^{2}(s) \hat{Z}_{s, \omega^{\prime}}\left(Z_{s, \omega^{\prime}}^{v}-\hat{Z}_{s, \omega^{\prime}}\right)+W^{2}(s) \hat{u}_{s, \omega^{\prime}}\left(v_{s, \omega^{\prime}}-\hat{u}_{s, \omega^{\prime}}\right)\right] d s+\mathrm{EE}^{\prime}\left[Q_{0}^{1} \hat{Y}_{0, \omega}\left(Y_{0, \omega}^{v}-\hat{Y}_{0, \omega}\right)+Q_{0}^{2} \hat{Y}_{0, \omega^{\prime}}\left(Y_{0, \omega^{\prime}}^{v}-\hat{Y}_{0, \omega^{\prime}}\right)\right]
\end{gathered}
$$

Applying Itô formulas to $p_{s, w}\left(Y_{s, \omega}^{v}-\hat{Y}_{s, \omega}\right)$ on $[0, T]$

$$
\begin{gathered}
\mathrm{EE}^{\prime}\left[Q_{0}^{1} \hat{Y}_{0, \omega}\left(Y_{0, \omega}^{v}-\hat{Y}_{0, \omega}\right)+Q_{0}^{2} \hat{Y}_{0, \omega^{\prime}}\left(Y_{0, \omega^{\prime}}^{v}-\hat{Y}_{0, \omega^{\prime}}\right)\right]=\mathrm{E} \int_{0}^{T} \mathrm{E}^{\prime}\left[C^{1}(s) p_{s, \omega}+F^{1}(s) q_{s, \omega}\right]\left(v_{s, \omega}-\hat{u}_{s, \omega}\right) d s \\
-\mathrm{E}_{0}^{T} \mathrm{E}^{\prime}\left[M^{1}(s) \hat{Y}_{s, \omega}\left(Y_{s, \omega}^{v}-\hat{Y}_{s, \omega}\right)+N^{1}(s) \hat{Z}_{s, \omega}\left(Z_{s, \omega}^{v}-\hat{Z}_{s, \omega}\right)\right] d s+\mathrm{E} \int_{0}^{T} \mathrm{E}^{\prime}\left[C^{2}(s)\left(v_{s, \omega^{\prime}}-\hat{u}_{s, \omega^{\prime}}\right) p_{s, \omega}+F^{2}(s)\left(v_{s, \omega^{\prime}}-\hat{u}_{s, \omega^{\prime}}\right) q_{s, \omega}\right] d s
\end{gathered}
$$$$
-\mathrm{E} \int_{0}^{T} \mathrm{E}^{\prime}\left[M^{2}(s) \hat{Y}_{s, \omega^{\prime}}\left(Y_{s, \omega^{\prime}}^{v}-\hat{Y}_{s, \omega^{\prime}}\right)+N^{2}(s) \hat{Z}_{s, \omega^{\prime}}\left(Z_{s, \omega^{\prime}}^{v}-\hat{Z}_{s, \omega^{\prime}}\right)\right] d s \text {. }
$$

We can get

$$
\begin{aligned}
& J(v)-J(\hat{u}) \geq \mathrm{E} \int_{0}^{T} \mathrm{E}^{\prime}\left[C^{1}(s) p_{s, \omega}+F^{1}(s) q_{s, \omega}\right]\left(v_{s, \omega}-\hat{u}_{s, \omega}\right) d s \\
& +\mathrm{E} \int_{0}^{T} \mathrm{E}^{\prime}\left[W^{1}(s) u_{s, \omega}\left(v_{s, \omega}-\hat{u}_{s, \omega}\right)+W^{2}(s) u_{s, \omega^{\prime}}\left(v_{s, \omega^{\prime}}-\hat{u}_{s, \omega^{\prime}}\right)\right] d s+\mathrm{E} \int_{0}^{T} \mathrm{E}^{\prime}\left[C^{2}(s)\left(v_{s, \omega^{\prime}}-\hat{u}_{s, \omega^{\prime}}\right) p_{s, \omega}+F^{2}(s)\left(v_{s, \omega^{\prime}}-\hat{u}_{s, \omega^{\prime}}\right) q_{s, \omega}\right] d s \\
& =\mathrm{E} \int_{0}^{T} \mathrm{E}^{\prime}\left[C^{1}(s) p_{s, \omega}+F^{1}(s) q_{s, \omega}\right]\left(v_{s, \omega}-\hat{u}_{s, \omega}\right) d s+\mathrm{E} \int_{0}^{T}\left[\left[\mathrm{E}^{\prime} W^{1}(s)\right] v_{s, \omega}\left(v_{s, \omega}-\hat{u}_{s, \omega}\right)+\mathrm{E}^{*}\left[W^{2}(s) \hat{u}_{s}\right]\left(v_{s, \omega}-\hat{u}_{s, \omega}\right)\right] d s \\
& +\mathrm{E} \int_{0}^{T} \mathrm{E}^{*}\left[C^{2}(s) p_{s}^{*}+F^{2}(s) q_{s}^{*}\right]\left(v_{s, \omega}-\hat{u}_{s, \omega}\right) d s
\end{aligned}
$$

Theorem 2 is verified. 


\section{Summary}

Theorem 1,(stochastic maximum principle) Assumed $(\hat{Y}(\cdot), \hat{Z}(\cdot), \hat{u}(\cdot))$ is the optimal trajectory of the control problem $\{(1),(2)\}, \forall v \in U$, a.e. $t \in[0, T]$, a.s.

$$
\left[\mathrm{E}^{\prime} \widehat{H}_{v}\left(t, \omega, \omega^{\prime}\right)+\mathrm{E}^{*} H_{v^{\prime}}\left(t, \omega^{*}, \omega\right)\right] \cdot(v-\hat{u}(t)) \geq 0
$$

where

$$
\widehat{H}\left(t, \omega, \omega^{\prime}\right)=H\left(t, \omega, \omega^{\prime}, \hat{Y}(t, \omega), \widehat{Z}(t, \omega), \hat{u}(t, \omega), \hat{Y}\left(t, \omega^{\prime}\right), \hat{Z}\left(t, \omega^{\prime}\right), \hat{u}\left(t, \omega^{\prime}\right), p(t, \omega), q(t, \omega)\right)
$$

Theorem 2. Assumes $\hat{u}$ that satisfy (9), the (p, g) is the solution of equation (10), the above LQ problem have unique solution.

\section{Acknowledgements}

This paper is funded by the project of National Natural Science Fund, Logistics distribution of artificial order picking random process model analysis and research (Project number: 71371033); and funded by intelligent logistics system Beijing Key Laboratory (No.BZ0211); and funded by scientific-research bases--- Science \& Technology Innovation Platform---Modern logistics information and control technology research (Project number: PXM2015_014214_000001); and funded by 2014-2015 school year, Beijing Wuzi University, College students' scientific research and entrepreneurial action plan project (No.68); and funded by Beijing Wuzi University, Yunhe scholars program(00610303/007); and funded by Beijing Wuzi University, Management science and engineering Professional group of construction projects. (No. PXM2015 014214 000039). University Cultivation Fund Project of 2014-Research on Congestion Model and algorithm of picking system in distribution center (0541502703)

\section{References}

[1] A. Szukala, A Knese-type theorem for euqation $x=f(t, x)$ in locally convex spaces, Journal for analysis and its applications, 18 (1999), 1101-1106.
[2] M. Tang and Q. Zhang, Optimal variational principle for backward stochastic control systems associated with Levy processes, Sci China Math, 55 (2012), 745-761.

[3] SevaS. Tang and X. Li, Necessary condition for optimal control of stochastic systems with random jumps, SIAM J Control Optim, 32 (1994), 1447-1475.

[4] J. Valero, On the kneser property for some parapolic problems, Topology and its applicanons, 155 (2005), 975-989.

[5] Z. Wu, Maximum principle for optimal control problem of fully coupled forward-backward stochastic systems, J. Systems Sci. Math. Sci., 11 (1998), 249-259.

[6] Z. Wu, Forward-backward stochastic differential equations with Brownian Motion and Process Poisson, Acta Math. Appl. Sinica, English Series, 15 (1999), 433-443.

[7] Z. Wu, A maximum principle for partially observed optimal control of forward-backward stochastic control systems, Sci China Ser F, 53 (2010), 2205-2214.

[8] Z. Wu and Z. Yu, Fully coupled forward-backward stochastic differential equations and related partial differential equations system, Chinese Ann Math Ser A, 25 (2004), 457-468

[9] H. Xiao and G. Wang, A necessary condition for optimal control of initial coupled forward-backward stochastic differential equations with partial information, J. Appl. Math. Comput., 37 (2011), 347-359.

[10] J. Xiong, An Introduction to Stochastic Filtering Theory, London, U.K.: Oxford University Press, 2008. 